326 Stohmann: Ueber den Wärmewerth der

\title{
Zehnte Abhandlung. \\ Ueber den Wärmewerth der Methylgruppen in den homologen Phenolen.
}

Vergleichen wir die in vorstehender Abhandlung nachgewiesenen Verbrennungswärmen der höheren Homologen des Phenols mit dem früher gefundenen Wärmewerth des Phenols ${ }^{1}$ ), so ergiebt uns die Differenz derselben den Werth der substituirenden Methylgruppen, oder den Werth der Gleichung: $\quad x=-\mathrm{H}+\mathrm{CH}_{3}$.

Da die Schmelzwärme des Phenols, nach den Untersuchungen von Pettersson ${ }^{2}$ ), 2343 cal. beträgt, so können wir den Wärmewerth der flüssigen Verbindung aus dem der festen ableiten, und somit die festen Körper mit festem und die flüssigen mit flüssigem Phenol vergleichen. Zum Vergleich mit dem Orcin haben wir das Resorcin, dessen Verbrennungswärme sich in unserer Arbeit über die Oxybenzole (siebente Abhandlung) findet. ${ }^{3}$ )

\section{Wärme- Diff. gegen Phenol von werth gleich. Aggregatzust. \\ cal. cal.}

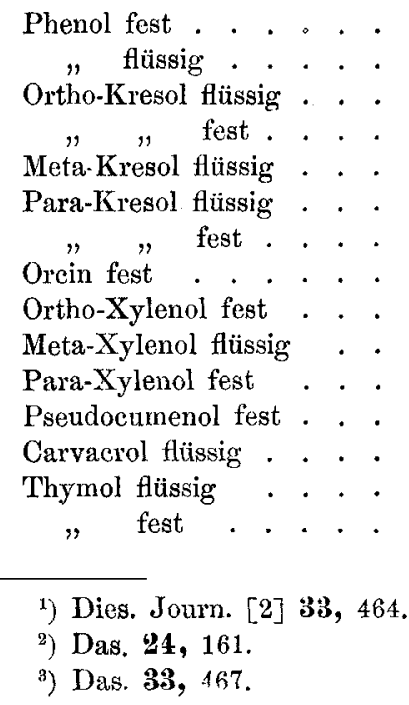

723659

726002

883008

879758

880956

882900

880441

824724

1035434

1037499

1035638

1191451

1354819

1353750

1349982

157006

156099

154954

156898

156782

153944

155888.2

155748.2

155989.2

155931.3

157204.4

156937.4

156581.4

Mittel 156356 
Methylgruppen in den homologen Phenolen.

Der Werth $x$ in obiger Gleichung wird daher:

$$
-\mathrm{H}+\mathrm{CH}_{3}=+156356 \mathrm{cal} \text {. }
$$

Dieser Werth stimmt aber völlig überein mit dem $W$ erth $156163 \mathrm{cal}$, welchen wir früher (fünfte Abhandlung) ${ }^{1}$ ) für die Homologen des Methylalkohols, auf Grundlage des Wärmewerthes der zugehörigen Säuren abgeleitet haben.

In den Phenolen, bis zu den Xylenolen, haben wir die Substitution der Methyle im Benzolkern. Da diese Substitution dieselbe Vermehrung des Wärmewerthes bedingt, wie die Substitution des Methyls in der Alkoholreihe, so ergiebt sich aber auch, dass diejenigen Isomere des Phenols, bei welchen die Substitution in den Seitenketten erfolgt ist, gleichen Wärmewerth besitzen müssen, wie diejenigen, bei denen die Substitution im Kern erfolgt ist. So müssen z. B. die Aethylphenole $\mathrm{C}_{k} \mathrm{H}_{4}<\mathrm{C}_{2} \mathrm{H}_{5}$ gleichen $W$ ärmewerth haben wie die Xylenole $\mathrm{C}_{6} \mathrm{H}_{3}(\mathrm{OH}) \backslash \mathrm{CH}_{3}$ u. s. w.

Die beiden letzten Glieder der obigen Phenolrcihe, das Carvacrol und das Thymol, unterscheiden sich von den vorhergehenden durch den Eintritt der Isopropylgruppe $-\mathrm{CH} \backslash \mathrm{CH}_{3}{ }_{\mathrm{CH}_{3}}^{\mathrm{CH}}$. Da hier dieselbe Wärmetönung erfolgt, wie beim Eintritt von drei einzelnen Methylgruppen in den Kern, und da diese gleich ist der Substitution in der normalen Reihe, so folgt hieraus auch, dass der Wärmewerth der Isoverbindungen dem der normalen Verbindungen gleich sein müsse.

Leipzig, im Juli 1886.

1) Dies. Journ. [2] 32, 420. 